\title{
The inhibitor of autophagy SBI-0206965 aggravates atherosclerosis through decreasing myeloid-derived suppressor cells
}

\author{
BO WANG ${ }^{1,2}$, GUANJUN DONG $^{2}$, QINGQIING ZHANG ${ }^{2}$, FENGLIAN YAN $^{2}$, ZHIHUA LI $^{2}$, \\ CHUNXIA LI ${ }^{2}$, HUI ZHANG ${ }^{2}$, QUN MA ${ }^{2}$, JUN DAI ${ }^{2}, \mathrm{CHUANPING} \mathrm{SI}^{2}$ and HUABAO XIONG ${ }^{3}$ \\ ${ }^{1}$ Institute of Forensic Medicine and Laboratory Medicine; ${ }^{2}$ Institute of Immunology and Molecular Medicine, \\ Jining Medical University, Jining, Shandong 272067, P.R. China; ${ }^{3}$ Department of Medicine, \\ Immunology Institute, Icahn School of Medicine at Mount Sinai, New York, NY 10029, USA
}

Received April 19, 2019; Accepted November 14, 2019

DOI: $10.3892 /$ etm.2019.8317

\begin{abstract}
Atherosclerosis (AS) is currently the leading cause of mortality worldwide, with the development of new strategies to prevent the formation and rupture of atherosclerotic plaques being a paramount area of research. Amounting evidence suggests autophagy has an important role in the pathogenesis of AS and may be a potential therapeutic target. In this study, the effect of SBI-0206965(6965), a novel inhibitor of autophagy, was tested on the development of AS in apolipoprotein E deficient $\left(\mathrm{ApoE}^{-/-}\right)$mice. Systemic application of 6965 was found to aggravate AS, with increased plaque size and decreased plaque stability in comparison with the control. Of note, it was observed that 6965 decreased the proportion of myeloid-derived suppressor cells (MDSCs). Further investigation demonstrated MDSCs markedly alleviated AS in $\mathrm{ApoE}^{-/-}$mice; while 6965 reduced the viability and promoted apoptosis of MDSCs in vitro. This is the first study describing an association between autophagy and MDSCs in AS models, providing a novel mechanism to potentially target in the management of this condition.
\end{abstract}

\section{Introduction}

Atherosclerosis (AS) is a chronic inflammatory disease characterized by the formation of plaques in the walls of large and medium-sized arteries (1). Unstable atherosclerotic plaques-especially those with scarce smooth muscle cells (SMCs) and increased inflammation and apoptosis-are closely related to numerous diseases, including acute coronary syndrome, myocardial infarction and stroke (2).

Correspondence to: Dr Huabao Xiong, Department of Medicine, Immunology Institute, Icahn School of Medicine at Mount Sinai, 1468 Madison Avenue, New York, NY 10029, USA

E-mail: huabao.xiong@mssm.edu

Key words: autophagy, SBI-0206965, atherosclerosis, plaque stability, myeloid-derived suppressor cells
Notwithstanding the numerous available therapeutic alternatives, AS remains the leading cause of mortality worldwide. Therefore, the development of novel strategies to prevent the formation and rupture of plaques has become a paramount area of research.

Macroautophagy (hereafter referred to as autophagy) is an evolutionarily conserved mechanism which eliminates various intracellular components such as damaged proteins, lipid droplets and organelles. Accumulating evidence suggests autophagy has an important role in the pathogenesis of AS and related disorders (3-5). Basal autophagy maintains cellular lipid homeostasis (6-9) and the induction of autophagy has been observed to prevent AS (10-13). However, previous research shows conflicting results concerning the impact of the inhibition of autophagy on AS with some studies indicating impaired autophagy promotes this process (14-18) and others suggesting otherwise. For example, Chen et al (19) found inhibition of autophagy in endothelial cells provided anti-atherosclerotic effects. Similarly, Dai et al (20) found 3-methyladenine (3-MA), a widely used inhibitor of autophagy, hindered the formation of atherosclerotic lesions and increased plaque stabilization. Further research is needed to elucidate the effects and molecular mechanisms of autophagy in AS, in order to explore potential therapeutic targets.

SBI-0206965 (hereafter referred to 6965) is a newly discovered inhibitor of the uncoordinated (Unc) 51-like kinase 1 (ULK1), which plays a pivotal role in autophagy. As the only conserved serine/threonine kinase in autophagy, ULK1 has become a very attractive target for therapeutic development (21). 6965 has also been observed to suppress autophagy induced by inhibition of mammalian target of rapamycin (22), inhibit AMP-activated protein kinase (23) and induce apoptosis (24). In one of the authors' previous studies (25), it was reported that 6965 regulated the functionality of granulocytic myeloid-derived suppressor cells (MDSCs), which have been linked to the development of AS (26).

The present study systemically administered 6965 to apolipoprotein $\mathrm{E}$ deficient $\left(\mathrm{ApoE}^{-/-}\right)$mice fed with a high-fat diet (HFD) and assessed its effect on AS. The current study found that 6965 promoted the formation of atherosclerotic lesions and 
reduced plaque stability. Interestingly, lower levels of cluster of differentiation (CD) $11 \mathrm{~b}^{+} \mathrm{Gr}-1^{+}$MDSCs were also detected following treatment with 6965. Subsequent in vitro experiments revealed 6965 reduced the viability and promoted the apoptosis of MDSCs in the presence of oxidized low-density lipoprotein (oxLDL). Adoptive transfer of MDSCs impaired the development of atherosclerotic plaques in $\mathrm{ApoE}^{-/}$mice. In consonance, these results suggest inhibition of autophagy by 6965 intervenes in the pathophysiology of AS by decreasing levels of MDSCs, outlining the mechanisms underlying the role of autophagy in this process.

\section{Materials and methods}

Animals. A total of $40 \mathrm{ApoE}^{-/-}$mice (19-21.5 g) were purchased from Beijing Vital River Laboratory Animal Technology Co., Ltd., and kept in a specific pathogen-free environment. All experiments were conducted according to the institutional guidelines for animal care and use, and ethical approval was obtained prior to the start of the study from an ethics committee of the Jining Medical University (approval number: 2019-FJ-002). The mice were housed at a constant temperature $\left(22^{\circ} \mathrm{C}\right)$ and relative humidity $40-70 \%$ under a $12 \mathrm{~h}$ dark/12 h light cycle. All mice had access to water and food ad libitum. To induce AS, 8-week old male $\mathrm{ApoE}^{-/}$mice were fed an HFD $(0.25 \%$ cholesterol and $15 \%$ cocoa butter) for 6 weeks. After one week of HFD, the mice were randomly divided into two groups ( $n=6$ per group): The 6965-treated group, which was injected intraperitoneally with 6965 (Selleck Chemicals), $150 \mu \mathrm{g} /$ mouse once per week for 4 weeks; and a control group of $\mathrm{ApoE}^{-/} \mathrm{AS}$ mice injected with PBS following the same pattern. One week after the last treatment, the mice were sacrificed and the tissues were harvested for further use. In the experiment with the adoptive transferring of MDSCs, mice were fed an HFD for 6 weeks. During this time, mice ( $n=6$ per group) received an intravenous injection of $6 \times 10^{6}$ MDSCs or PBS every 10 days in the experimental and control groups, respectively.

Serum cholesterol levels. Blood samples (110 $\mu 1$ per mouse) were collected by retro-orbital bleed following general anesthesia (2-4\% ether inhalation anesthesia) according to ethical approvals $(27,28)$. After $30 \mathrm{~min}$ of incubation at room temperature, samples were centrifuged at $2,775 \mathrm{x} \mathrm{g}$ at room temperature for $10 \mathrm{~min}$ to obtain serum, which was stored at $-80^{\circ} \mathrm{C}$ until analysis. Total plasma cholesterol and total triglyceride levels were determined with automated enzymatic techniques (7080; Hitachi, Ltd.).

Histopathology and immunohistochemistry (IHC). After sacrificing, mice were perfused with PBS via the left cardiac ventricle and their hearts and aortic roots were harvested, fixed in $4 \%$ paraformaldehyde at room temperature overnight and embedded with paraffin. Serial paraffin sections of 4-6 $\mu \mathrm{m}$ were dissected longitudinally. At least three sections were obtained from each aortic root, with $50 \mu \mathrm{m}$ of separation between each other. After staining with hematoxylin and eosin (H\&E) for 15 and 5 min, respectively (both at room temperature), sections were blocked using 5\% goat serum (Beijing Solarbio Science \& Technology Co., Ltd.) at room temperature for $30 \mathrm{~min}$. Corresponding sections on separate sliders were then stained for various anti- bodies at $37^{\circ} \mathrm{C}$ for $1 \mathrm{~h}$. The antibodies used included Anti-F4/80 (1:400; cat. no. ab111101; Abcam), rabbit anti- $\alpha$ smooth muscle actin (1:200; cat. no. bs-10196R; Biosynthesis Biotechnology Co., Ltd.) or anti-mouse Gr-1 (1:50; cat. no. 108436; Biolegend). The Rabbit two-step test kit (cat. no. PV-9001; Origene Technologies, Inc.) was used for the secondary antibody binding procedure, and the staining with secondary antibodies was performed at $37^{\circ} \mathrm{C}$ for $15 \mathrm{~min}$, according to the manufacturer's protocol. Terminal deoxynucleotidyl transferase-mediated dUTP nick end labelling (TUNEL) was performed on paraffin sections with a TUNEL kit (Roche Diagnostics) following the manufacturer's protocol in order to assess apoptosis. Images were captured using an Olympus fluorescence/light microscope (IX71; Olympus Corporation) and positive staining was quantified by computer-assisted histomorphometry (Image-Pro Plus 6.0, Media Cybernetics, Inc.). To evaluate vascular obstruction, the ratio of total plaque area to aortic root cross sectional area was calculated. For each IHC analysis, it was calculated as the percentage of immunostained area in relation to the total area. Mean values were calculated from the corresponding three consecutive sections from each mouse.

Flow cytometry. After sacrificing the mice, blood, bone marrow and spleen cells were harvested from each group $(n=6$ per group). Red blood cells were removed using erythrocyte lysis buffer [Multi Sciences (Lianke) Biotech, Co., Ltd.]. Then, the remaining cells were washed with PBS and resuspended in proportions of $1 \times 10^{6}$ cells per ml. Fluorescently labeled antibodies against CD11b (FITC-labeled) and Gr-1 (APC-labeled) were purchased from Biolegend and used at a 1:100 dilution to detect MDSCs $\left(\mathrm{CD} 11 \mathrm{~b}^{+}, \mathrm{Gr}-1^{+}\right)$. Flow cytometry was also performed to detect the apoptosis of cells stained with the Annexin V-PI kit (Annexin V-FITC Apoptosis Detection kit; Biouniquer) according to the manufacturer's protocol.

Adoptive transfer of MDSCs. Bone marrow-derived MDSCs were obtained as described previously (25). In brief, bone marrow cells were isolated from the tibias and femurs of $\mathrm{ApoE}^{-/-}$ mice. Then, these were cultured in complete DMEM, which was supplemented and stimulated with combinations of granulocyte macrophage colony stimulating factor (GM-CSF) $(40 \mathrm{ng} / \mathrm{ml}$, PeproTech, Inc.) and interleukin (IL)-6 (40 ng/ml, PeproTech, Inc.). The cultures were maintained during 4 days in an atmosphere humidified with $5 \% \mathrm{CO}_{2}$ at $37^{\circ} \mathrm{C}$ with the objective of inducing MDSCs. The experimental group of $\mathrm{ApoE}^{-/-}$mice which underwent adoptive transfer $(n=6)$ received MDSCs suspended in PBS via intravenous injection. Each animal received $6 \times 10^{6}$ cells every 10 days during 6 weeks. The control group was injected with PBS only following the same schedule.

En face staining analysis. After the mice were sacrificed, the aortas were harvested from heart to the iliac arteries. Then, they were fixed in $4 \%$ paraformaldehyde overnight at room temperature and stained with $0.5 \%$ Oil Red O (O0625; Sigma-Aldrich; Merck KGaA) for $12 \mathrm{~h}$ at $4^{\circ} \mathrm{C}$ using a light microscope.

CCK-8 assay. Cell viability was tested using Cell Counting Kit-8 (CCK-8) assays (HY-K0301; MedChemExpress) according to the manufacturer's protocols. Mouse MDSCs 
A
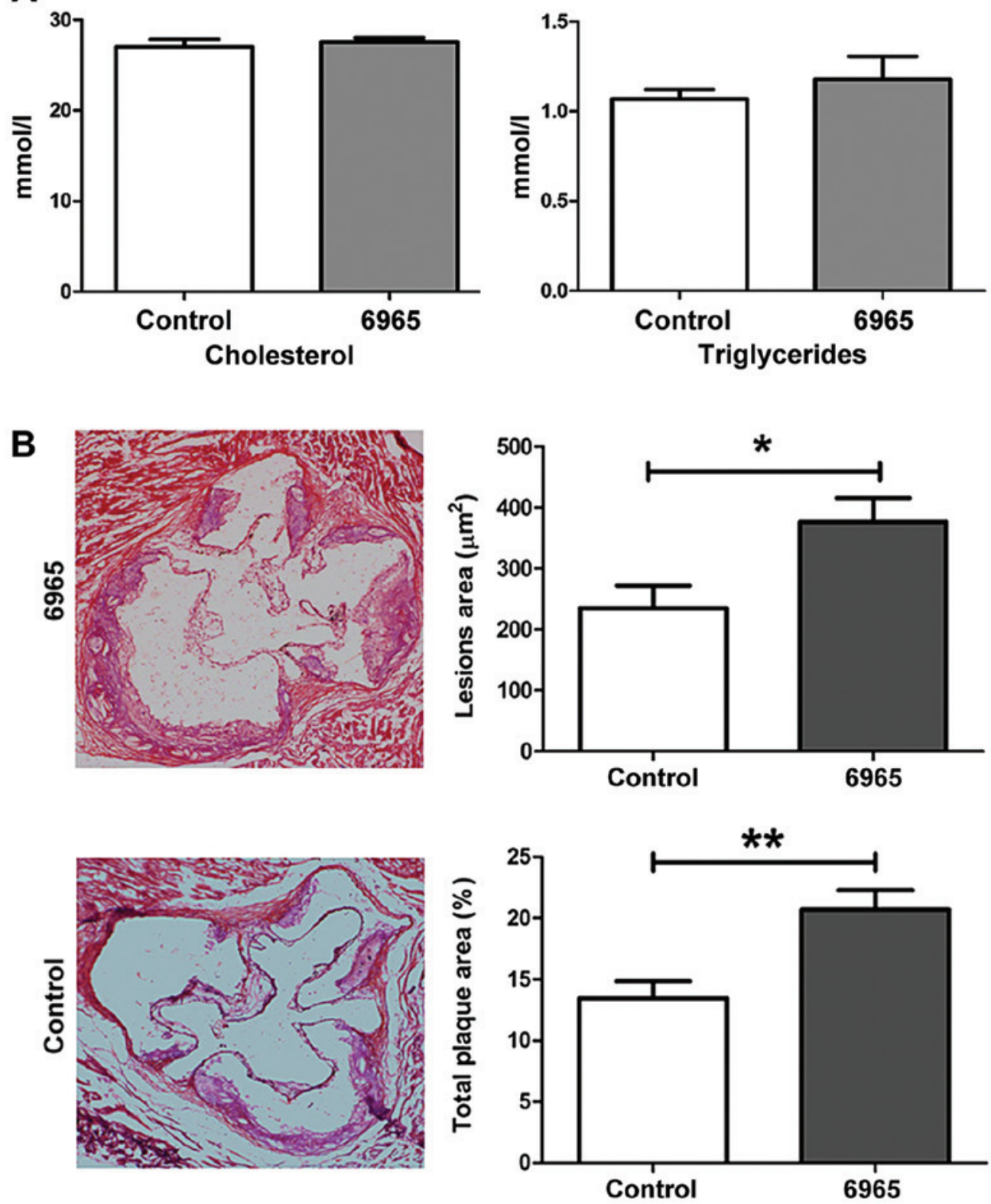

Figure 1. Treatment with 6965 promotes the development of atherosclerotic lesions independent of cholesterol levels. (A) Total plasma cholesterol and triglyceride levels of the control and 6965 groups. (B) Representative hematoxylin and eosin staining depicting the aortic roots from mice in the control and 6965-treated groups (magnification, $\mathrm{x} 20$ ). Lesion area $\left(\mu \mathrm{m}^{2}\right)$ represents the sum of all the areas occupied by plaques in every pathological section. Total plaque area (\%) represents the ratio of total plaque area to the cross-sectional area of the aortic root. Data are shown as the mean \pm standard deviation. "P<0.05 and ${ }^{* *} \mathrm{P}<0.01$ vs. the control.

were seeded in 96-well cell culture plates at $5 \times 10^{3}$ cells per well and divided into two groups: One subjected to oxLDL (25 $\mu \mathrm{g} / \mathrm{ml})$ treatment only, and one preincubated with 6965 $(10 \mu \mathrm{M})$ for $30 \mathrm{~min}$ before treatment with oxLDL. Next, the cells were cultured at $37^{\circ} \mathrm{C}$ for 24,48 and $72 \mathrm{~h}$ respectively, and then incubated with $10 \mu \mathrm{k}$ CCK- 8 solution at $37^{\circ} \mathrm{C}$ for $1 \mathrm{~h}$. Well absorbance was set to $450 \mathrm{~nm}$. Each treatment was performed in triplicate.

Statistical analysis. Data were expressed as the mean \pm standard deviation. Unpaired Student's t-test was used to compare data between different groups. Results were considered significant when $\mathrm{P}<0.05$. Statistical analysis was performed with GraphPad Prism 5.0 software (GraphPad Software, Inc.).

\section{Results}

6965 increases the size of atherosclerotic plaques independent of lipid levels. To investigate the effects of 6965 on AS, eight-week old male ApoE $\mathrm{E}^{--}$mice were fed an HFD for 6 weeks total in order to induce AS. After one week of HFD, the 6965-treated group was injected intraperitoneally with 6965 for 4 weeks. One week after the last treatment, the mice were sacrificed and analyzed. As shown in Fig. 1A, 6965 did not affect the lipid profile, including total cholesterol and triglyceride levels. H\&E staining revealed 6965 administration was associated with significantly greater plaque size on aortic roots and obvious damage to the adventitia $(\mathrm{P}<0.05$; Fig. $1 \mathrm{~B})$. To assess vascular obstruction, the ratio of total plaque area to 

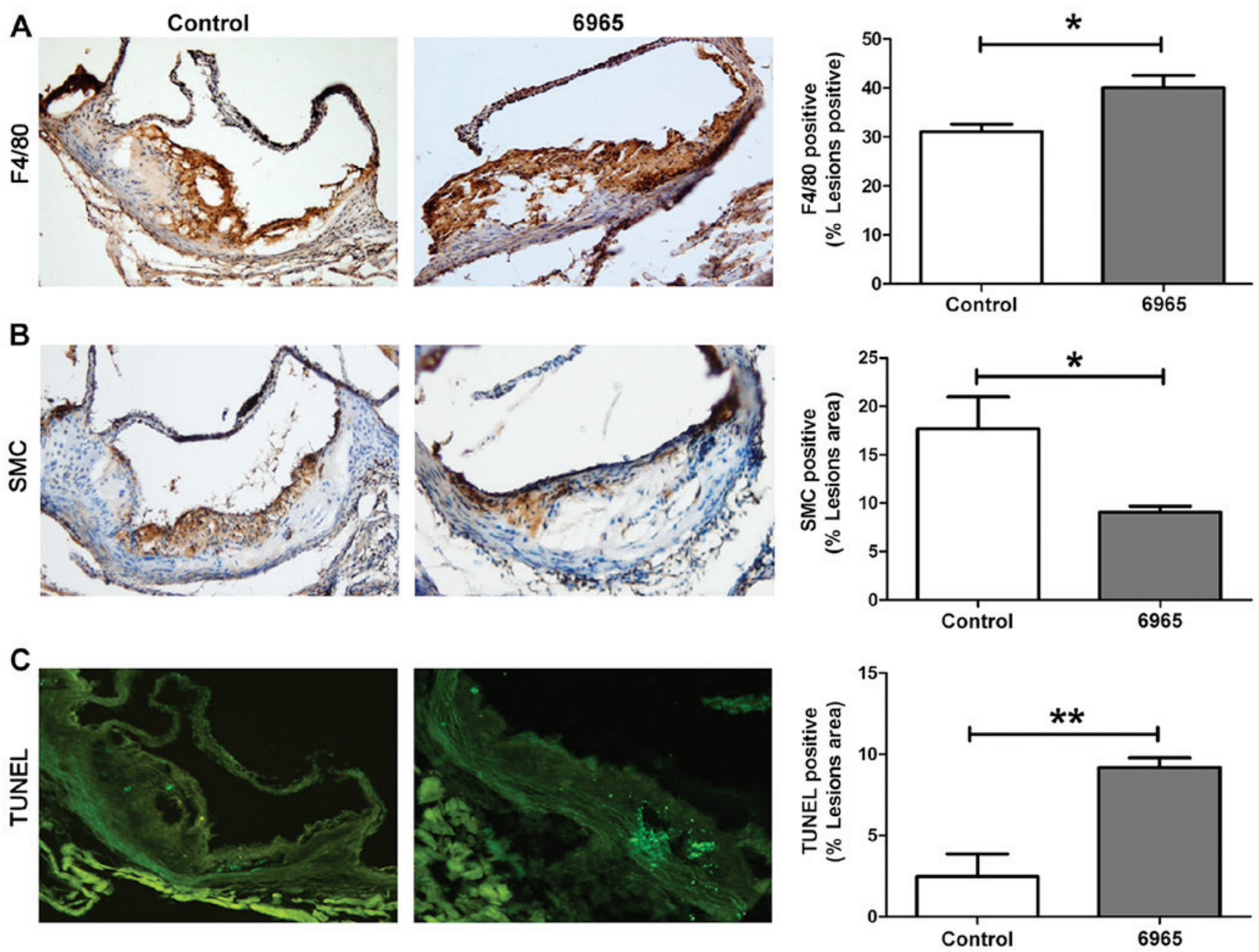

Figure 2. Treatment with 6965 decreases plaque stability. Representative images of (A) macrophages, (B) smooth muscle cells and (C) apoptosis from the control and 6965-treated groups (magnification, x40). Macrophage and SMC contents were analyzed through immunohistochemistry. TUNEL staining was used to detect apoptotic cells. Data are presented as the percentage of stained plaque areas in the entirety of the lesions. Data are shown as the mean \pm standard deviation. ${ }^{*} \mathrm{P}<0.05$ and ${ }^{* *} \mathrm{P}<0.01$ vs. the control. TUNEL, Terminal deoxynucleotidyl transferase-mediated dUTP nick end labelling; SMC, smooth muscle cell.

aortic root cross-sectional area was measured, and it was found that treatment with 6965 was related to significant increases in this parameter in comparison with the controls. Therefore, the present study surmised that 6965 increased aortic obstruction and aggravated AS independently of cholesterol and triglyceride levels.

6965 decreases the stability of atherosclerotic plaques. Plaque stability rather than size may be more important in the development of complications associated with AS. In order to evaluate the effects of 6965 on plaque stability, IHC staining was implemented to observe the proportions of SMC and infiltrated macrophages in local lesions; whereas the TUNEL method was used to assess levels of apoptosis. As shown in Fig. 2, 6965 significantly increased the proportions of macrophages that presented as F4/80 and decreased the proportion of SMC in plaques $(\mathrm{P}<0.05)$. In addition, 6965 significantly increased the number of TUNEL-positive apoptotic cells $(\mathrm{P}<0.01)$. These results indicate treatment with 6965 reduced plaque stability.

6965 decreases the levels of MDSCs in atherosclerosis models with $\mathrm{ApoE}^{-/-}$mice. A recent study reported that $\mathrm{CD} 11 \mathrm{~b}^{+} \mathrm{Gr}-1^{+}$ MDSCs reduce atherosclerotic plaques in $\mathrm{LDLr}^{-/-}$mice (26), suggesting these cells play an important role in the pathophysiology of AS. In this study, the proportions of $\mathrm{CD}_{11 \mathrm{~b}^{+}}$ Gr- $1^{+}$MDSCs in peripheral blood, spleen and bone marrow were determined by flow cytometry. The present study found treatment with 6965 significantly decreased the proportion of $\mathrm{CD}_{11 \mathrm{~b}}{ }^{+} \mathrm{Gr}-1^{+} \mathrm{MDSC}$ in comparison with controls. This was the case in the bone marrow, the spleen and the peripheral blood ( $\mathrm{P}<0.05$; Fig. 3A). IHC staining of the lesions showed Gr- ${ }^{+}$levels were also significantly decreased in 6965-treated mice $(\mathrm{P}<0.05$; Fig. 3B). This suggests 6965 intervenes in the pathogenesis of AS by lowering the levels of $\mathrm{CD} 11 \mathrm{~b}^{+} \mathrm{Gr}-1^{+} \mathrm{MDSCs}$.

Adoptive transfer of MDSCs impairs the development of atherosclerotic plaques in ApoE $E^{-/-}$mice. To assess the role of MDSCs in AS, an adoptive transfer of MDSCs into ApoE ${ }^{-/-}$mice was performed. MDSCs were isolated from the bone marrow of $\mathrm{ApoE}^{-/-}$mice without HFD as described previously (25), and then injected intravenously in $\mathrm{ApoE}^{-/-}$atherosclerotic models. As expected, a significant reduction of atherosclerotic lesions in MDSCs-treated mice in both the aorta $(\mathrm{P}<0.05$; Fig. $4 \mathrm{~A})$ and aortic root $(\mathrm{P}<0.05$; Fig. $4 \mathrm{~B})$ in comparison with the controls was observed, which did not undergo adoptive transfer. This confirms the protective effect of MDSCs on AS in $\mathrm{ApoE}^{-/-}$mice. 
A
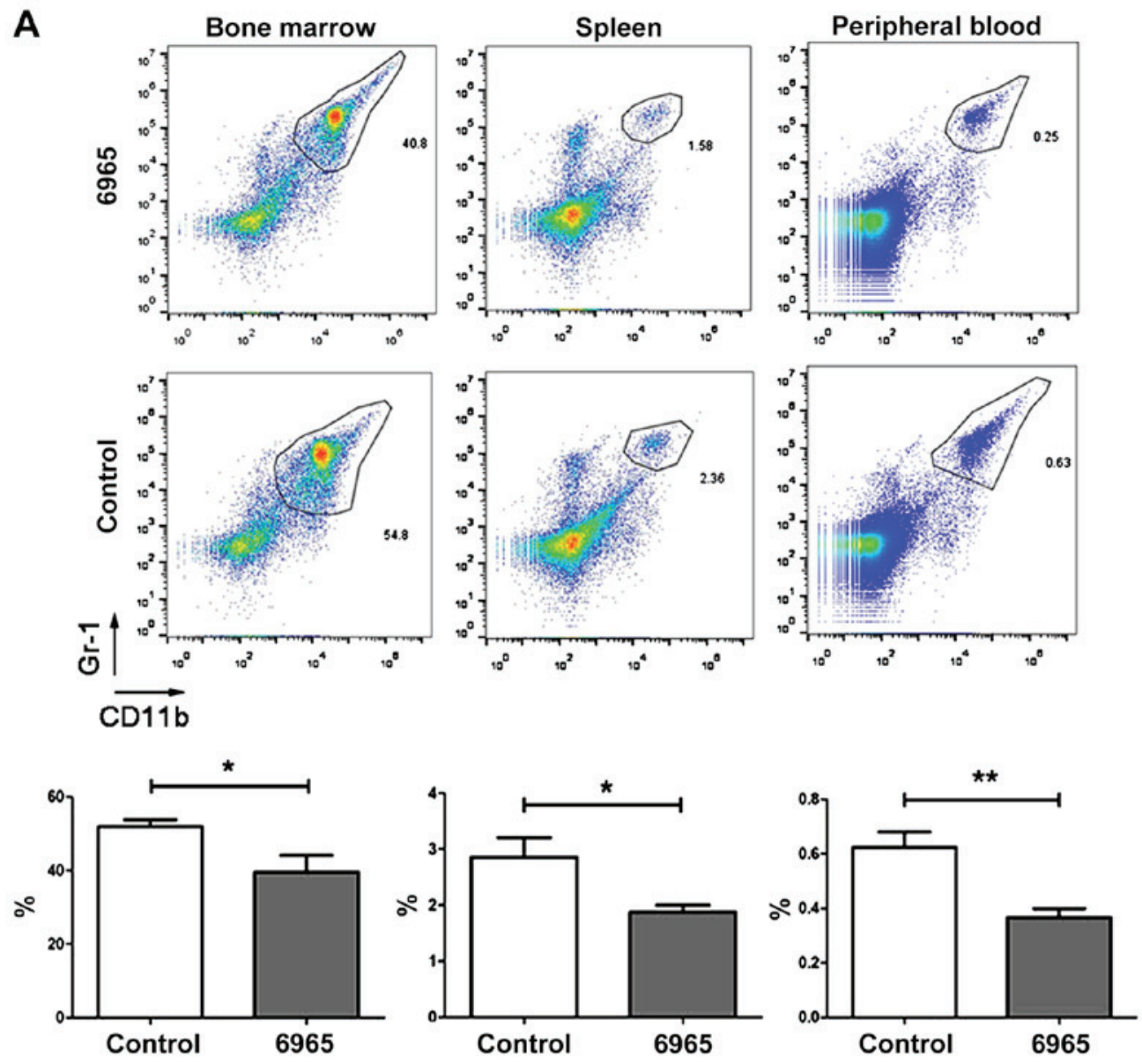

B
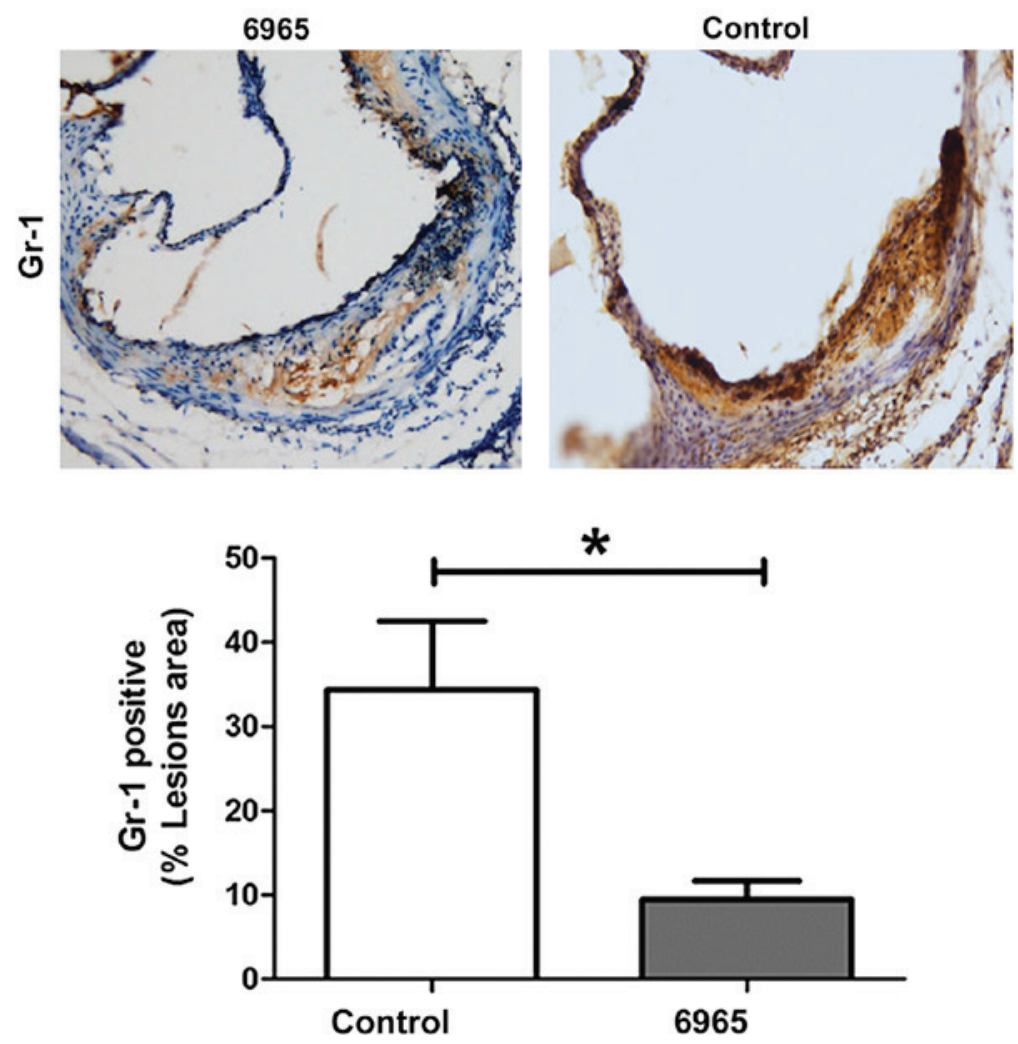

Figure 3. Treatment with 6965 decreases the levels of MDSCs. (A) Flow cytometry and fluorescent staining were used to evaluate the proportions of CD11b $\mathrm{Gr}-1^{+}$MDSCs. Representative images of the bone marrow, spleen and peripheral blood from different groups are shown. Data are presented as the mean \pm SD. ${ }^{*} \mathrm{P}<0.05$ and ${ }^{* *} \mathrm{P}<0.01$. (B) The expression of $\mathrm{Gr}-1$ in plaques was analyzed by immunohistochemistry and the percentage of Gr-1 positive area was calculated in the total lesioned area (magnification, $\mathrm{x} 40$ ). Data are shown as the mean $\pm \mathrm{SD}$. " $\mathrm{P}<0.05$ vs. the control. SD, standard deviation; $\mathrm{CD}$, cluster of differentiation; MDSCs, myeloid-derived suppressor cells. 
A
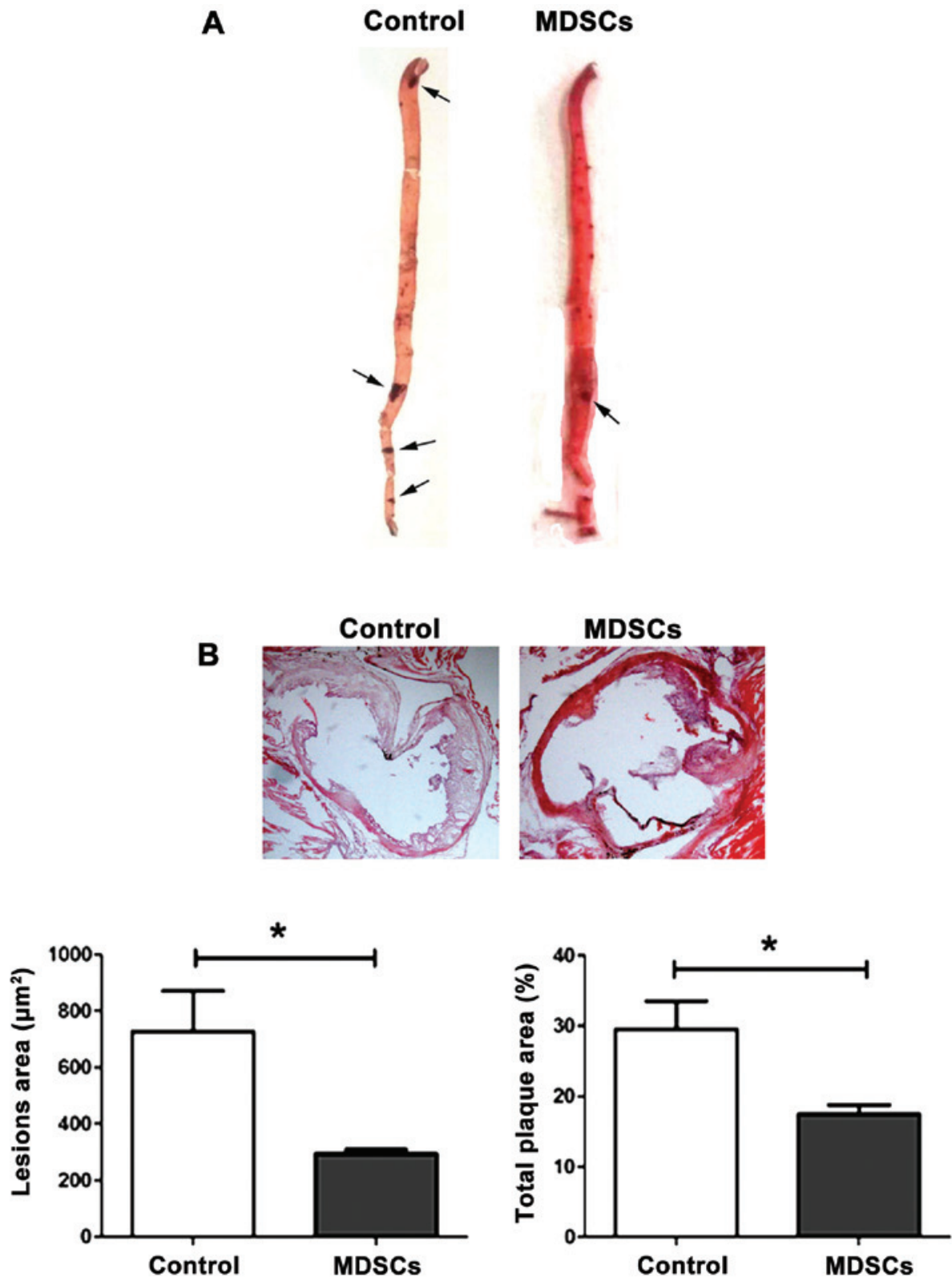

Figure 4. Adoptive transfer of MDSCs reduces atherosclerotic lesions in $\mathrm{ApoE}^{-/}$mice. (A) Representative images of oil red O-stained plaques in entire aortas (thoracic aorta and abdominal aorta) from the control and MDSC adoptive transfer groups (magnification, x10). The black arrow indicates where the plaques are. (B) Representative cross-sections of lesions in the aortic valve area stained with hematoxylin and eosin are shown. Lesion area $\left(\mu \mathrm{m}^{2}\right)$ represents the sum of all the areas occupied by plaques in every pathological section (magnification, $\mathrm{x} 20$ ). Total plaque area (\%) represents the ratio of total plaque area to the cross-sectional area of the aortic root. Data are shown as the mean \pm standard deviation. ${ }^{*} \mathrm{P}<0.05$ vs. the control. MDSCs, myeloid-derived suppressor cells; $\mathrm{ApoE}^{--}$, apolipoprotein E deficient.

6965 reduces the viability and increases the apoptosis of oxLDL-stimulated MDSCs. In order to investigate the impact of 6965 on MDSCs in AS, 6965-treated MDSCs from C57 mice were exposed to oxLDL in vitro. Assessment of CCK-8 levels revealed 6965 reduced MDSCs viability in the presence of oxLDL (Fig. 5A). Cell Annexin V-PI staining showed 6965 significantly increased the proportion of apoptosis in the presence of oxLDL in comparison with exposure to oxLDL alone $(\mathrm{P}<0.001$; Fig. 5B).

\section{Discussion}

Modulation of autophagy is an attractive potential alternative for the treatment of AS; therefore, it is a priority to focus research on the molecular mechanisms underlying this relationship in order to assess the feasibility and clinical applicability of autophagy-regulating agents. In this study, 6965, a novel inhibitor of ULK1, was found to enhance the formation of atherosclerotic lesions and reduced plaque stability; reflected in increased plaque size, reduced SMC content, and increased proportion of macrophage infiltration and apoptosis. Although a few studies have associated inhibition of autophagy with anti-atherosclerotic capacity $(19,20)$, most studies harmonize with the present conclusions, where inhibition of autophagy results in aggravation of AS. The conflicting results may be due to the heterogeneous modes of action of the various molecules implemented to inhibit autophagy in previous studies. In this study, 6965, a highly selective inhibitor of ULK1 was used. 
A

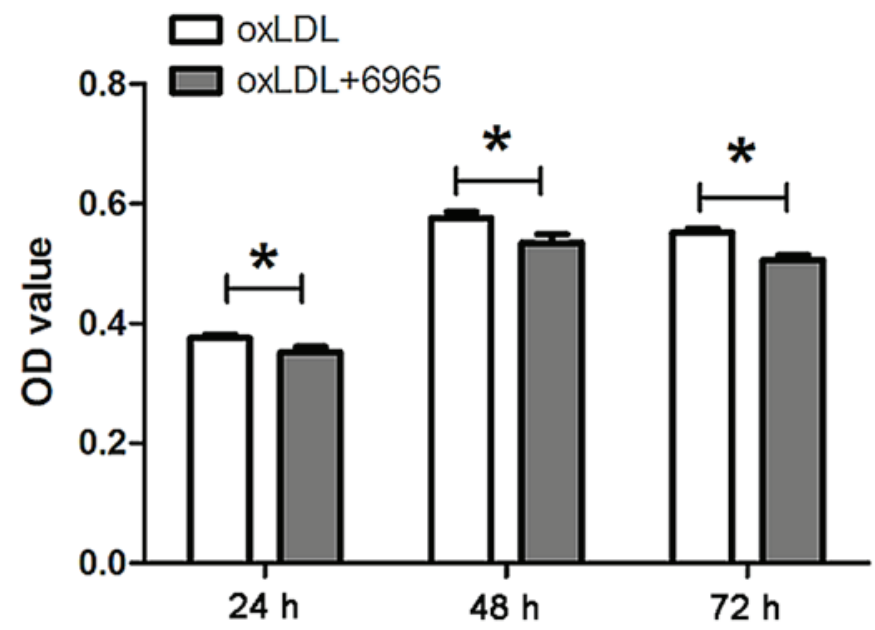

B

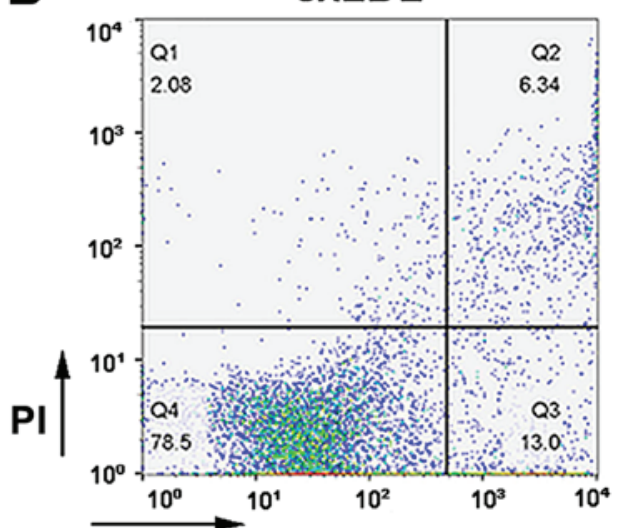

Annexin V

\section{oxLDL}

$48 \mathrm{~h}$
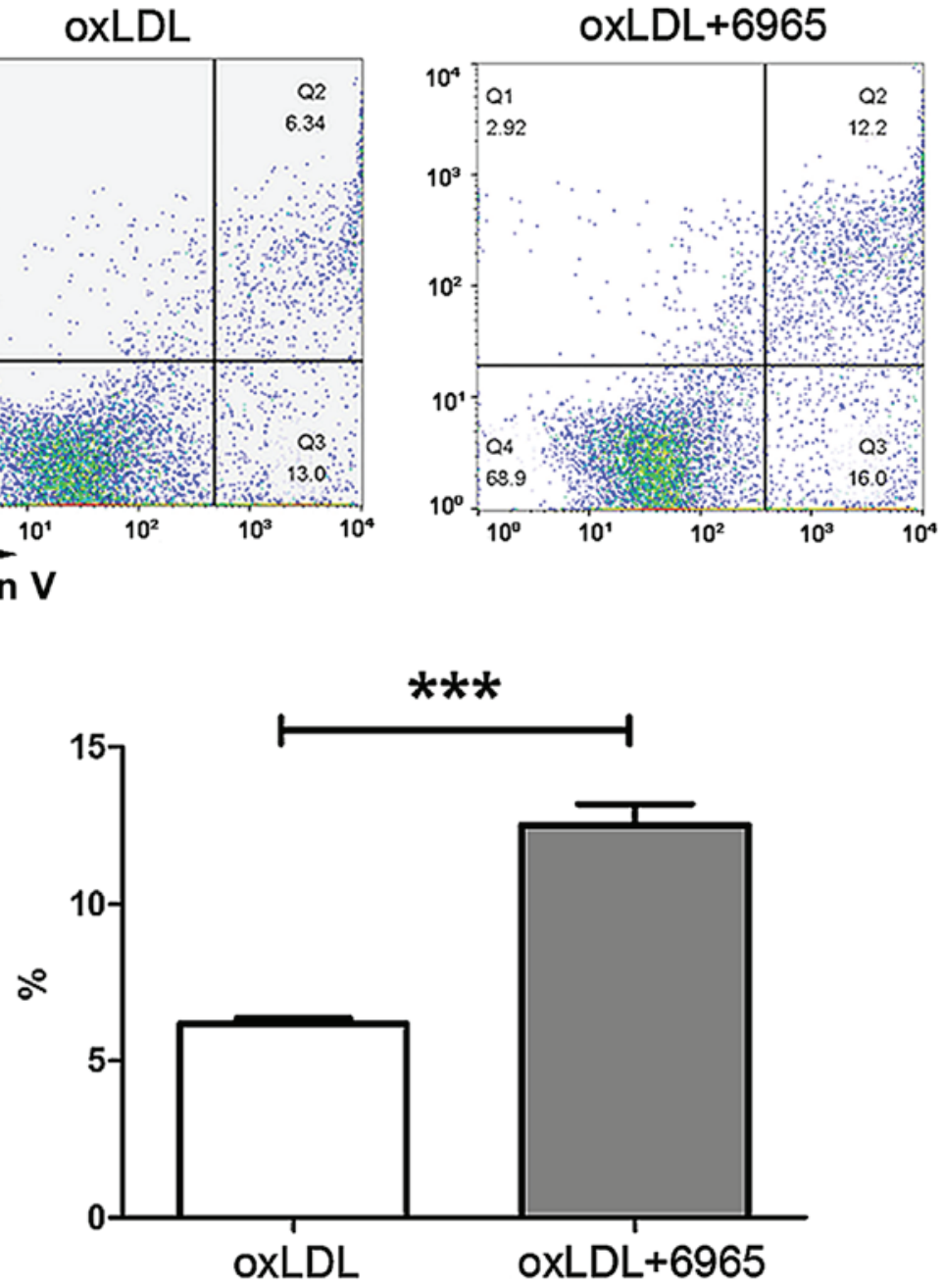

Figure 5. Treatment with 6965 decreases viability and increases apoptosis in oxLDL-stimulated MDSCs. (A) Cells were preincubated with 6965 (10 $\mu \mathrm{M})$ for $30 \mathrm{~min}$ and then stimulated with $25 \mu \mathrm{g} / \mathrm{ml}$ oxLDL. Cell Counting Kit-8 assays were performed at different times after oxLDL stimulation to measure MSDC viability. (B) Flow cytometry detected MDSCs apoptosis. Data are shown as means \pm standard deviation, calculated from triplicate experiments. " $\mathrm{P}<0.05$ and ${ }_{* * * *} \mathrm{P}<0.001$ vs. oxLDL. oxLDL, oxidized low density lipoprotein; MDSCs, myeloid-derived suppressor cells; PI, propidium iodide; OD, optical density.

This kinase is one of the most upstream components and the only serine/threonine kinase in the core autophagy pathway, representing an excellent drug target.

Notably, the proportion of $\mathrm{CD} 11 \mathrm{~b}^{+} \mathrm{Gr}-1^{+} \mathrm{MDSCs}$ were also found to be decreased after treatment with 6965. MDSCs are a heterogeneous population of cells, composed of early myeloid progenitor cells and immature myeloid cells. MDSCs can block hematoprogenitor cell differentiation and play a critical role in suppressing immune responses. Previous research has demonstrated that MDSCs are important immunosuppres- 
sive mediators in cancer and inflammatory disorders (29-34), including endotoxin shock, experimental autoimmune encephalomyelitis, graft-vs.-host disease, rheumatoid arthritis and diabetes. Recently, Foks et al (26) showed MDSCs reduced AS via suppression of pro-inflammatory immune responses in LDLr-deficient mice. To verify the effect of MDSCs on $\mathrm{ApoE}^{-/-}$ AS models, MDSCs were transplanted into $\mathrm{ApoE}^{-/-}$mice, which resulted in amelioration of the atherosclerotic plaques. In the present study, it was found that treatment with 6965 was associated with a decrease in MDSCs levels. Considering MDSCs mainly migrate to inflamed tissue, $\mathrm{Grl}^{+}$proportions were detected in atherosclerotic lesions and confirmed the decrease of MDSCs in local plaques in 6965-treated mice. Thus, the decrease of MDSCs may be a key mechanism underlying the of pro-atherosclerotic effects of 6965.

Numerous studies have identified autophagy as a major regulator of MDSC viability and function. Wu et al (35) demonstrated that the expression of autophagy marker LC3B and p62 was positively associated with MDSCs number. Parker et al (36) found that autophagy inhibitor chloroquine or bafilomycin reduced MDSCs viability. However, in another research, 3-MA did not induce MDSC-like cell model J774M cell death (37). In the authors' previous publication (25), inhibition of autophagy was found to contribute to the accumulation of granulocytic MDSCs in the context of endotoxin shock. In the present study, the influence of 6965 on MDSCs in the presence of oxLDL was appraised. The present results showed inhibition of autophagy by 6965 decreased MSDC viability and increased their apoptosis when exposed to oxLDL. It suggested that autophagy might regulate cell survival depending on the cellular context. Various conditions and effector molecules linking to autophagy are known to facilitate MDSC survival. For example, endoplasmic reticulum stress (ER stress) regulates MDSC half-life by controlling TRAIL receptor mediated apoptosis (38) and a novel study found that autophagy protected human umbilical vein endothelial cells against ER stress-mediated apoptosis (39). It is suggested that inhibition of autophagy might induce MDSC apoptosis through interruption of ER stress pathways. Moreover, increased apoptosis and impaired degradation capacity of lysosomes was observed in tumor-infiltrating autophagy deficient monocytic-MDSCs (40) which indicates that interruption of autophagy flux at the late stage induces cell death. Cytokines and chemokines, such as GM-CSF and IL-6, contributed to the expansion and accumulation of MDSCs (41). In the authors' previous study, 6965 treatment reduced the levels of IL-6, IL-12, tumor necrosis factor- $\alpha$ and CXCL9 in mice, which suggests that the influence of cytokines of 6965 might also affect the viability of MDSCs. Although there are numerous possible mechanisms, an accurate regulatory pathway remains to be determined.

In conclusion, 6965 may aggravate AS by reducing MDSCs functionality with a decrease in cell viability and the promotion of apoptosis being key related mechanisms. The present findings confirm the importance of autophagy in the pathophysiology of AS and highlight MDSCs as potential therapeutic targets in this condition.

\section{Acknowledgements}

Not applicable.

\section{Funding}

The present study was supported by the Doctoral Fund of Jining Medical University (grant no. 600353002) and the Teacher Research Support Fund of Jining Medical University (grant no. JYFC2018KJ058).

\section{Availability of data and materials}

The datasets used or analyzed during the current study are available from the corresponding author on reasonable request.

\section{Authors' contributions}

BW and $\mathrm{HX}$ designed the experiments. BW, QZ, HZ, ZL and CL performed animal experiments. GD performed flow cytometry. QZ and FY performed animal feeding, weighed mice and injected drugs. JD and BW performed cell culture and CCK-8 assays. CS and QM analyzed the data. BW wrote the manuscript. FY performed animal feeding and en face staining. All authors read and approved the final version of the manuscript.

\section{Ethics approval and consent to participate}

The research program was approved by the Ethics Committee of the Jining Medical University.

\section{Patient consent for publication}

Not applicable.

\section{Competing interests}

The authors declare that they have no competing interests.

\section{References}

1. Lusis AJ: Atherosclerosis. Nature 407: 233-241, 2000.

2. Zhu Y, Xian X, Wang Z, Bi Y, Chen Q, Han X, Tang D and Chen R: Research progress on the relationship between atherosclerosis and inflammation. Biomolecules 8: pii: E80, 2018.

3. Jiang Q, Hao R, Wang W, Gao $H$ and Wang C: SIRT1/Atg5/autophagy are involved in the antiatherosclerosis effects of ursolic acid. Mol Cell Biochem 420: 171-184, 2016.

4. Mialet-Perez $\mathrm{J}$ and Vindis C: Autophagy in health and disease: Focus on the cardiovascular system. Essays Biochem 61: 721-732, 2017.

5. Bravo-San Pedro JM, Kroemer G and Galluzzi L: Autophagy and mitophagy in cardiovascular disease. Circ Res 120: 1812-1824, 2017.

6. Sasaki Y, Ikeda Y, Iwabayashi M, Akasaki Y and Ohishi M: The impact of autophagy on cardiovascular senescence and diseases. Int Heart J 58: 666-673, 2017.

7. Grootaert MOJ, Roth L, Schrijvers DM, De Meyer GRY and Martinet W: Defective autophagy in atherosclerosis: To die or to senesce? Oxid Med Cell Longev 2018: 7687083, 2018.

8. Vion AC, Kheloufi M, Hammoutene A, Poisson J, Lasselin J, Devue C, Pic I, Dupont N, Busse J, Stark K, et al: Autophagy is required for endothelial cell alignment and atheroprotection under physiological blood flow. Proc Natl Acad Sci USA 114: E8675-E8684, 2017.

9. Sergin I, Evans TD, Zhang X, Bhattacharya S, Stokes CJ, Song E, Ali S, Dehestani B, Holloway KB, Micevych PS, et al: Exploiting macrophage autophagy-lysosomal biogenesis as a therapy for atherosclerosis. Nat Commun 8: 15750, 2017.

10. Evans TD, Jeong SJ, Zhang X, Sergin I and Razani B: TFEB and trehalose drive the macrophage autophagy-lysosome system to protect against atherosclerosis. Autophagy 14: 724-726, 2018. 
11. Lazaro I, Lopez-Sanz L, Bernal S, Oguiza A, Recio C, Melgar A, Jimenez-Castilla L, Egido J, Madrigal-Matute J and Gomez-Guerrero C: Nrf2 activation provides atheroprotection in diabetic mice through concerted upregulation of antioxidant, anti-inflammatory, and autophagy Mechanisms. Front Pharmacol 9: 819, 2018

12. Li X, Zhou Y, Zhang X, Cao X, Wu C and Guo P: Cordycepin stimulates autophagy in macrophages and prevents atherosclerotic plaque formation in $\mathrm{ApoE}^{-/}$mice. Oncotarget 8: 94726-94737, 2017.

13. Tang F and Yang TL: MicroRNA-126 alleviates endothelial cells injury in atherosclerosis by restoring autophagic flux via inhibiting of PI3K/Akt/mTOR pathway. Biochem Biophys Res Commun 495: 1482-1489, 2018.

14. Yang X, Wei J, He Y, Jing T, Li Y, Xiao Y, Wang B, Wang W, Zhang J and Lin R: SIRT1 inhibition promotes atherosclerosis through impaired autophagy. Oncotarget 8: 51447-51461, 2017.

15. Li W, Sultana N, Siraj N, Ward LJ, Pawlik M, Levy E, Bengtsson E and Yuan XM: Autophagy dysfunction and regulatory cystatin $\mathrm{C}$ in macrophage death of atherosclerosis. J Cell Mol Med 20: $1664-1672,2016$

16. Grootaert MO, da Costa Martins PA, Bitsch N, Pintelon I, De Meyer GR, Martinet W and Schrijvers DM: Defective autophagy in vascular smooth muscle cells accelerates senescence and promotes neointima formation and atherogenesis. Autophagy 11: 2014-2032, 2015.

17. Osonoi Y, Mita T, Azuma K, Nakajima K, Masuyama A, Goto H, Nishida Y, Miyatsuka T, Fujitani Y, Koike M, et al: Defective autophagy in vascular smooth muscle cells enhances cell death and atherosclerosis. Autophagy 14: 1991-2006, 2018.

18. Ouimet M, Ediriweera H, Afonso MS, Ramkhelawon B Singaravelu R, Liao X, Bandler RC, Rahman K, Fisher EA, Rayner KJ, et al: microRNA-33 regulates macrophage autophagy in atherosclerosis. Arterioscler Thromb Vasc Biol 37: 1058-1067, 2017.

19. Chen PS, Wang KC, Chao TH, Chung HC, Tseng SY, Luo CY, Shi GY, Wu HL and Li YH: Recombinant thrombomodulin exerts anti-autophagic action in endothelial cells and provides anti-atherosclerosis effect in apolipoprotein E deficient mice. Sci Rep 7: 3284, 2017

20. Dai S, Wang B, Li W, Wang L, Song X, Guo C, Li Y, Liu F, Zhu F, Wang Q, et al: Systemic application of 3-methyladenine markedly inhibited atherosclerotic lesion in $\mathrm{ApoE}^{-/}$mice by modulating autophagy, foam cell formation and immune-negative molecules. Cell Death Dis 7: e2498, 2016.

21. Zachari M and Ganley IG: The mammalian ULK1 complex and autophagy initiation. Essays Biochem 61: 585-596, 2017.

22. Egan DF, Chun MG, Vamos M, Zou H, Rong J, Miller CJ, Lou HJ, Raveendra-Panickar D, Yang CC, Sheffler DJ, et al: Small molecule inhibition of the autophagy kinase ULK1 and identification of ULK1 substrates. Mol Cell 59: 285-297, 2015.

23. Dite TA, Langendorf CG, Hoque A, Galic S, Rebello RJ, Ovens AJ, Lindqvist LM, Ngoei KRW, Ling NXY, Furic L, et al: AMP-activated protein kinase selectively inhibited by the type II inhibitor SBI-0206965. J Biol Chem 293: 8874-8885, 2018.

24. Tang F, Hu P, Yang Z, Xue C, Gong J, Sun S, Shi L, Zhang S, Li Z, Yang C, et al: SBI0206965, a novel inhibitor of Ulk1, suppresses non-small cell lung cancer cell growth by modulating both autophagy and apoptosis pathways. Oncol Rep 37: 3449-3458, 2017.

25. Dong G, Si C, Zhang Q, Yan F, Li C, Zhang H, Ma Q, Dai J, Li Z, Shi H, et al: Autophagy regulates accumulation and functional activity of granulocytic myeloid-derived suppressor cells via STAT3 signaling in endotoxin shock. Biochim Biophys Acta 1863: 2796-2807, 2017.

26. Foks AC, Van Puijvelde GH, Wolbert J, Kröner MJ, Frodermann V, Van Der Heijden T, Van Santbrink PJ, Boon L, Bot I and Kuiper J: CD11b ${ }^{+} \mathrm{Gr}-1^{+}$myeloid-derived suppressor cells reduce atherosclerotic lesion development in LDLr deficient mice. Cardiovasc Res 111: 252-261, 2016.
27. Nakatsu N, Igarashi Y, Aoshi T, Hamaguchi I, Saito M, Mizukami T, Momose H, Ishii KJ and Yamada H: Isoflurane is a suitable alternative to ether for anesthetizing rats prior to euthanasia for gene expression analysis. J Toxicol Sci 42: 491-497, 2017.

28. Akeju O, Hamilos AE, Song AH, Pavone KJ, Purdon PL and Brown EN: GABAA circuit mechanisms are associated with ether anesthesia-induced unconsciousness. Clin Neurophysiol 127: 2472-2481, 2016

29. Shaikh F, He J, Bhadra P, Chen X and Siu SWI: TNF receptor type II as an emerging drug target for the treatment of cancer, autoimmune diseases, and graft-vs.-host disease: Current perspectives and in silico search for small molecule binders. Front Immunol 9: 1382, 2018.

30. Boros P, Ochando J and Zeher M: Myeloid derived suppressor cells and autoimmunity. Hum Immunol 77: 631-636, 2016.

31. Shi H, Dong G, Yan F, Zhang H, Li C, Ma Q, Zhang J, Ning Z, Li Z, Dai J, et al: Arctigenin Ameliorates inflammation by regulating accumulation and functional activity of MDSCs in endotoxin shock. Inflammation 41: 2090-2100, 2018.

32. Elliott DM, Singh N, Nagarkatti $M$ and Nagarkatti PS: Cannabidiol attenuates experimental autoimmune encephalomyelitis model of multiple sclerosis through induction of myeloid-derived suppressor cells. Front Immunol 9: 1782, 2018.

33. Casacuberta-Serra S, Costa C, Eixarch H, Mansilla MJ, López-Estévez S, Martorell L, Parés M, Montalban X, Espejo C and Barquinero J: Myeloid-derived suppressor cells expressing a self-antigen ameliorate experimental autoimmune encephalomyelitis. Exp Neurol 286: 50-60, 2016.

34. Hsieh CC, Lin CL, He JT, Chiang M, Wang Y, Tsai YC, Hung $\mathrm{CH}$ and Chang PJ: Administration of cytokine-induced myeloid-derived suppressor cells ameliorates renal fibrosis in diabetic mice. Stem Cell Res Ther 9: 183, 2018.

35. Wu JS, Li L, Wang SS, Pang X, Wu JB, Sheng SR, Tang YJ, Tang YL, Zheng M and Liang XH: Autophagy is positively associated with the accumulation of myeloid-derived suppressor cells in 4-nitroquinoline-1-oxide-induced oral cancer. Oncol Rep 40: 3381-3391, 2018

36. Parker KH, Horn LA and Ostrand-Rosenberg S: High-mobility group box protein 1 promotes the survival of myeloid-derived suppressor cells by inducing autophagy. J Leukoc Biol 100: 463-470, 2016

37. Liu F, Li X, Lu C, Bai A, Bielawski J, Bielawska A, Marshall B, Schoenlein PV, Lebedyeva IO and Liu K: Ceramide activates lysosomal cathepsin B and cathepsin D to attenuate autophagy and induces ER stress to suppress myeloid-derived suppressor cells. Oncotarget 7: 83907-83925, 2016.

38. Condamine T, Kumar V, Ramachandran IR, Youn JI, Celis E, Finnberg N, El-Deiry WS, Winograd R, Vonderheide RH, English NR, et al: ER stress regulates myeloid-derived suppressor cell fate through TRAIL-R-mediated apoptosis. J Clin Invest 124: 2626-2639, 2014.

39. Li CF, Pan YK, Gao Y, Shi F, Wang YC and Sun XQ: Autophagy protects HUVECs against ER stress-mediated apoptosis under simulated microgravity. Apoptosis 24: 812-825, 2019.

40. Alissafi T, Hatzioannou A, Mintzas K, Barouni RM, Banos A, Sormendi S, Polyzos A, Xilouri M, Wielockx B, Gogas H and Verginis P: Autophagy orchestrates the regulatory program of tumor-associated myeloid-derived suppressor cells. J Clin Invest 128: 3840-3852, 2018.

41. Tamadaho RSE, Hoerauf A and Layland LE: Immunomodulatory effects of myeloid-derived suppressor cells in diseases: Role in cancer and infections. Immunobiology 223: 432-442, 2018.

This work is licensed under a Creative Commons Attribution-NonCommercial-NoDerivatives 4.0 International (CC BY-NC-ND 4.0) License. 